\title{
Mycoplasma pneumoniae may cause dyspnoea and hospitalisations in young healthy adults
}

\author{
Riku Metsälä ${ }^{1}$ Solja Ala-Korpi ${ }^{1}$ Juha Rannikko ${ }^{1,2} \cdot$ Merja Helminen $^{3}$ - Marjo Renko ${ }^{4,5}$ (D)
}

Received: 25 August 2020 / Accepted: 24 January 2021 / Published online: 3 February 2021

(C) The Author(s) 2021

\begin{abstract}
Polymerase chain reaction (PCR)-based diagnostics for Mycoplasma pneumoniae (M. pneumoniae) from the respiratory tract has become widely available, but the interpretation of the results remains unclear. M. pneumoniae has been suggested to cause mainly mild and self-limiting infections or asymptomatic carriage. However, systematic analyses of the association between PCR results and clinical findings are scarce. This study aimed to clarify the clinical features of PCR-positive M. pneumoniae infections in a hospital setting. We reviewed 103 PCR-positive patients cared for in a university hospital during a 3-year period. Data on age, sex, health condition, acute symptoms, other pathogens found, laboratory and X-ray results and treatments were collected. Over $85 \%$ of the patients had a triad of typical symptoms: fever, cough and shortness of breath. Symptoms in the upper respiratory tract were rare. In $91 \%$ of the cases, M. pneumoniae was the only pathogen found. The highest incidence was found in the age group of 30-40 years, and 68\% of the patients did not have any underlying diseases. Most patients were initially empirically treated with beta-lactam antibiotics and needed 2-4 changes in their treatment. Only $6 \%$ were discharged without an antibiotic effective against M. pneumoniae. This study shows that M. pneumoniae often led to hospitalisation and that patients needed appropriate antimicrobial treatment to recover. Mixed infections were rare, and situations that could be interpreted as carriage did not occur.
\end{abstract}

Keywords Community-acquired pneumonia $\cdot$ Shortness of breath $\cdot$ Respiratory infection $\cdot$ Clinical features $\cdot$ Mycoplasma pneumoniae

\section{Introduction}

Mycoplasma pneumoniae (M. pneumoniae, mycoplasma) is one of the smallest bacteria in terms of cellular dimensions and genome [1]. M. pneumoniae typically infects children and young adults [2], and it has been suggested to mainly cause mild and self-limiting respiratory tract infections usually managed by primary health care [3]. M. pneumoniae is also capable of causing community-acquired pneumonias (CAP) and

Marjo Renko

marjo.renko@uef.fi

1 Faculty of Medicine and Health Technology, Tampere University, Tampere, Finland

2 Tampere University Hospital, Tampere, Finland

3 Tampere Center for Child Health Research, Tampere, Finland

4 Department of Pediatrics, University of Eastern Finland and Kuopio University Hospital, Box 100, 70029 Kuopio, Finland

5 PEDEGO Research Unit, University of Oulu, Oulu, Finland epidemics in schools, camps, military bases and other communities [4, 5]. Although the majority of mycoplasma infections are mild, severe respiratory manifestations may require intensive care unit admissions [6]. Extrapulmonary manifestations, such as Stevens-Johnson syndrome, pericarditis or encephalitis, are also possible [7].

In the past, the lack of quick and efficient microbiological methods made the clinical diagnosis of $M$. pneumonia infections challenging [8]. Although nucleic acid tests, such as polymerase chain reaction (PCR), have become widely available, the role of M. pneumoniae in the aetiology of respiratory infections remains unclear. Studies using PCR tests have shown that M. pneumoniae is carried at relatively high rates in the respiratory tract of healthy children [1]. Conversely, infection may lead to intensive care in adults [3]. Randomised trials on treatment have not been published [9], and it has been argued whether M. pneumoniae is a real pathogen requiring treatment.

To clarify the role of $M$. pneumoniae in respiratory infections, we evaluated the clinical course of PCR-positive M. pneumoniae infections in a hospital setting. We also assessed the frequency of spontaneous recovery and the 
proportion of mixed infections in these mycoplasma PCRpositive patients.

\section{Materials and methods}

We performed a retrospective case analysis of M. pneumoniae PCR-positive patients at the Tampere University Hospital, Finland. The Tampere University Hospital serves an area of $530,000[10]$ inhabitants as both a secondary and a tertiary referral hospital. Multiplex PCR, which recognises M. pneumoniae, Chlamydia pneumoniae, Legionella pneumophila, Bordetella pertussis and Bordetella parapertussis, was introduced in March 2014 in our hospital (Seegene Anyplex ${ }^{\mathrm{TM}}$ II RB5, South Korea and Bio-Rad CFX96, USA). The results of the test were available for clinicians usually in one working day. We collected data on consecutive patients who had multiplex PCR taken from a nasopharyngeal swab sample between March 2014 and February 2017. The medical records of $M$. pneumoniae PCR-positive patients were reviewed. Only community-acquired infections were included.

We recorded the data on age, sex, underlying diseases, acute symptoms, diagnoses and duration of hospital stay; the highest values of inflammatory parameters during hospital stay (C reactive protein [CRP] and leucocytes); results of all microbial tests available (e.g. blood cultures and tests for respiratory viruses); thoracic X-rays; and antimicrobial treatments. Antimicrobial treatments were classified based on their efficacy against $M$. pneumoniae. Macrolides, doxycycline, tetracyclines and fluoroquinolones were considered effective treatments [4]. Data on acute symptoms, such as fever, cough, rhinitis and shortness of breath, were recorded based on medical records either as the patients' subjective experience or the clinicians' assessment. If the symptoms were not mentioned as present or absent, the data were recorded as missing.

The multiplex PCR test for respiratory viruses included adenovirus; influenza A and B viruses; parainfluenza virus types 1-4; rhino-, respiratory syncytial-, boca- and metapneumoviruses; coronavirus types 229E, NL63 and

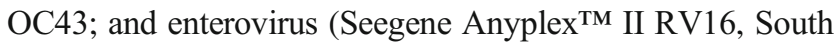
Korea and Bio-Rad CFX96, USA). Blood cultures were collected in BD BACTEC blood culture bottles and placed in the automated microbial detection system BD BACTEC FX (Franklin Lakes, NJ, USA).

M. pneumoniae serology or culture of the swabs was not systematically performed.

In the statistical analyses, we calculated the proportions for the dichotomous variables and the medians and ranges for the continuous variables. The proportion differences between the two groups with $95 \%$ confidence intervals were computed with the standard normal deviate test (Stats Direct 3.0). The age distribution of the patients was plotted in 10-year age groups. The time of year of the infections was plotted by summarising the cases in all 3 years in each month.

\section{Results}

Respiratory bacterial multiplex PCR was taken from 1,309 patients (1,190 adults and 119 children) during the 3-year study period. M. pneumoniae PCR was positive in $103(8 \%)$ cases, the majority of which were males $(59,57 \%)$. The median age of the PCR-positive patients was 39 years (range 3.687.9) (Table 1). The number of PCR tests taken was the highest in the age groups of $60-80$ years and $0-10$ years, but the incidence of positive results was the highest in the age group of 30-40 years (Fig. 1). Most of the cases were detected from September to November (Fig. 2). Three patients were discharged from the emergency room, while the remaining patients needed hospital care for a median duration of 5 days (range 1-32 days) (Table 1). Five (5\%) patients were admitted to the intensive care unit. Any kind of underlying disease was present in 30 (29\%) patients (Table 1). The multiplex PCR test was taken on admission or during the first day of care in $70(68 \%)$ patients.

The most common symptoms were cough in 99 (99\%) patients, fever $\geq 38^{\circ} \mathrm{C}$ in $87(85 \%)$ and shortness of breath

Table 1 Basic characteristics of the 103 patients with a positive M. pneumoniae PCR in a hospital-based setting

\begin{tabular}{ll}
\hline Characteristic & $N=103$ \\
\hline Males, $N(\%)$ & $59(57.3 \%)$ \\
Age (years), mean (SD), [range] & $39.9(17.9),[3.6-87.9]$ \\
Fever $>38{ }^{\circ} \mathrm{C}, N(\%)$ & $87(84.5 \%)$ \\
Cough, $N(\%)$ & $99(99 \%)$ \\
Shortness of breath, $N(\%)$ & $85(88.5 \%)$ \\
Rhinitis, $N(\%)$ & $14 / 63(22.2 \%)$ \\
Max CRP, median [range] & $142[1.9-449]$ \\
Max leukocytes, median [range] & $11[1.6-63]$ \\
Pneumonia in chest radiograph, $N(\%)$ & $92(91.1 \%)$ \\
Pleural fluid in chest radiograph, $N(\%)$ & $9(8.9 \%)$ \\
Length of hospital stay (days), median [range] & $5[0-32]$ \\
Underlying diseases, yes (\%) & $30(29.1 \%)$ \\
- Asthma, $N(\%)$ & $8(27 \%)$ \\
- Cardiac disease, $N(\%)$ & $7(23 \%)$ \\
- Malignancy, $N(\%)$ & $3(10 \%)$ \\
- Hypertension, $N(\%)$ & $3(10 \%)$ \\
- COPD, $N(\%)$ & $2(7 \%)$ \\
- Other, $N(\%)$ & $10(33 \%)$ \\
Epilepsy $(3)$, rheumatism (2), HIV (1), & \\
anaemia (1), hyponatremia $(1)$, & \\
hypothyroidism (1), juvenile idiopathic & \\
arthritis $(1)$ & \\
\hline
\end{tabular}


Fig. 1 Age distribution of the 103 patients with a positive or negative $M$. pneumoniae PCR tests in a hospital-based setting

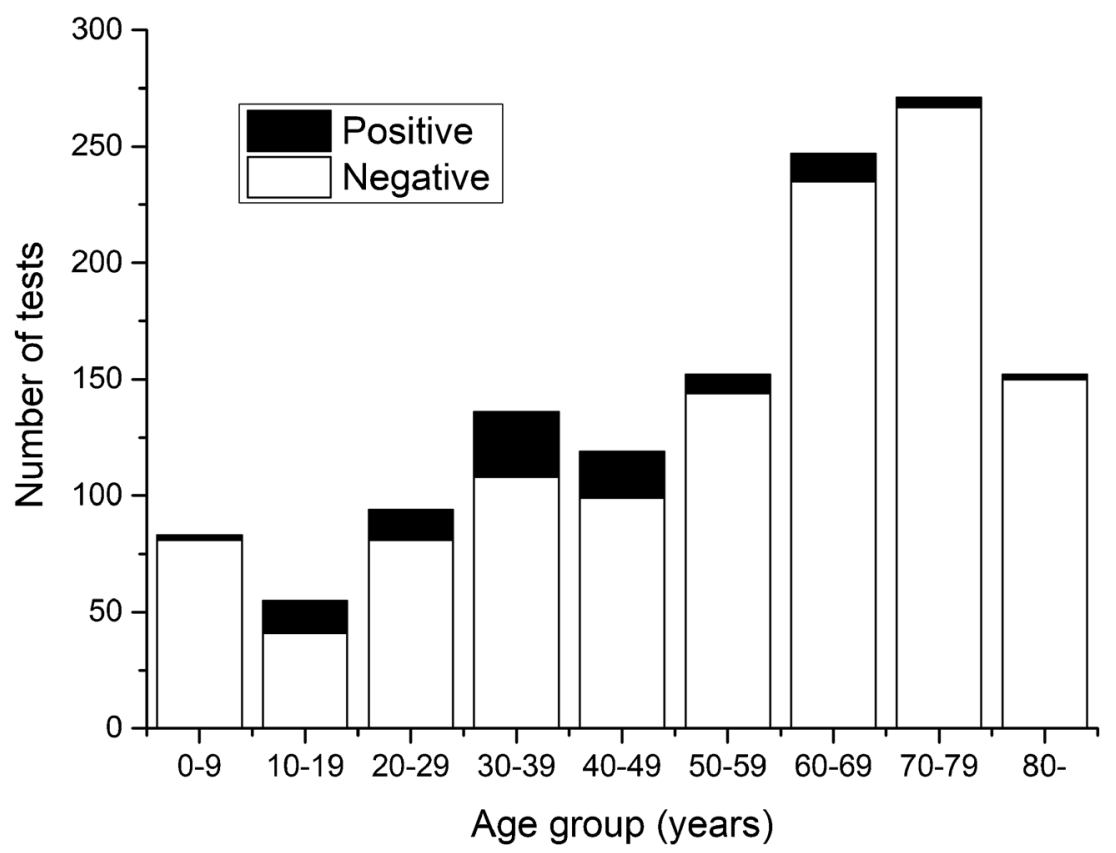

in $85(89 \%)$. Information on the upper respiratory tract symptoms was available in $63(61 \%)$ patients, and $14(22 \%)$ of them had rhinitis (Table 1). The acute symptoms did not differ between previously healthy patients and those with underlying diseases (Table 2). Three patients, two children and one adult, had symptoms and signs compatible with StevensJohnson syndrome. Thoracic X-ray was performed in 101 (98\%) cases, and $94(91 \%)$ images showed signs of pneumonia and $9(9 \%)$ of pleural fluid (Table 1).

None of the patients had other bacteria aside from M. pneumoniae in the multiplex PCR. Blood culture was performed in 95 (92\%) patients and 94 of them were negative. One child had a blood culture positive for Streptococcus pneumoniae. Multiplex PCR for respiratory viruses was obtained from $88(85 \%)$ patients, and positive results were found in eight patients, including rhino- (4), boca- (2), corona- (1) and human metapneumoviruses (1). Influenza or respiratory syncytial viruses were not detected.

All 103 patients were treated with antimicrobial agents. In almost all patients $(102,99 \%)$, one or more antibiotic changes were made during hospitalisation. Ten (10\%) patients received treatment effective against $M$. pneumoniae immediately on
Fig. 2 Number of monthly admissions of patients with a positive M. pneumoniae PCR test in a hospital-based setting

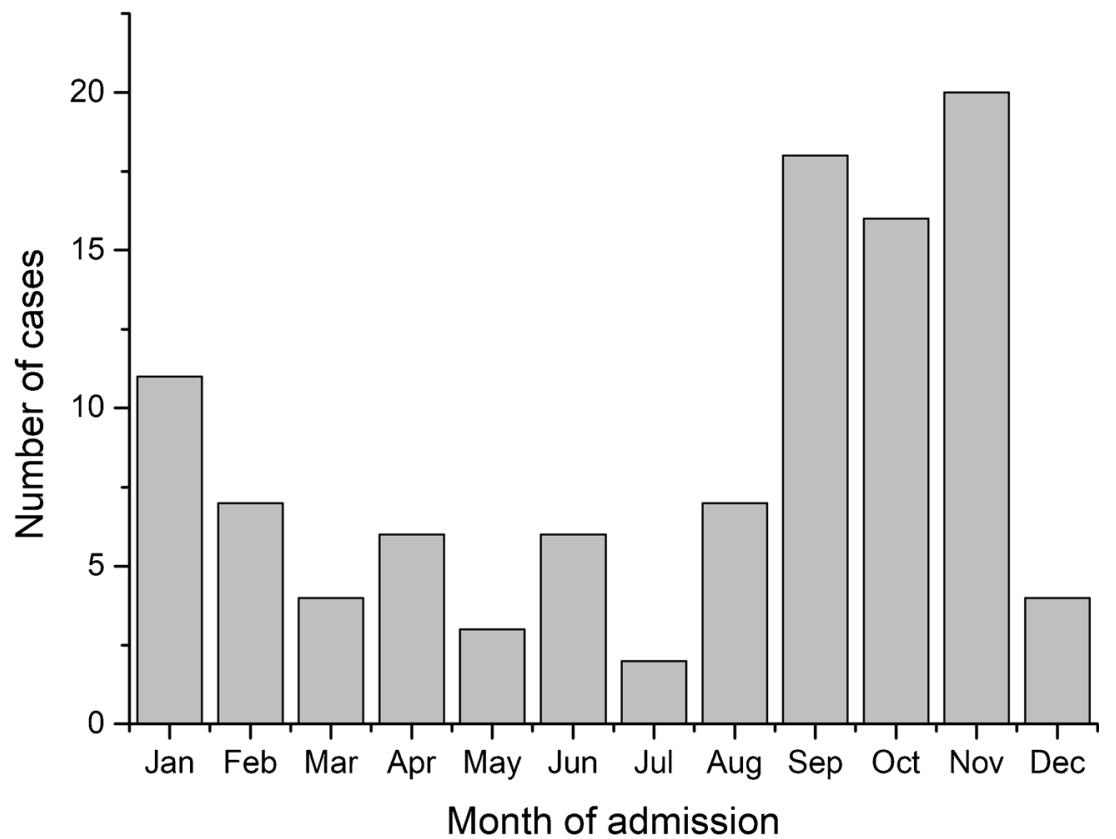


Table 2 Symptoms of M. pneumoniae infection in 73 previously healthy patients and 30 patients with some underlying diseases

\begin{tabular}{lllll}
\hline & Healthy $N=73$ & Underlying disease $N=30$ & Difference (95\% CI) & $P$ value \\
\hline Fever $38{ }^{\circ} \mathrm{C}, N(\%)$ & $64(88 \%)$ & $23(77 \%)$ & $11 \%(-4$ to 30$)$ & 0.15 \\
Cough, $N(\%)$ & $71(97 \%)$ & $28(93 \%)$ & $4 \%(-4$ to 19$)$ & 0.33 \\
Shortness of breath, $N(\%)$ & $59(81 \%)$ & $26(87 \%)$ & $-5 \%(-20$ to 12$)$ & 0.42 \\
Rhinitis, $N(\%)$ & $9 / 45(20 \%)$ & $5 / 18(28 \%)$ & $-8 \%(-33$ to 13$)$ & 0.36 \\
\hline
\end{tabular}

admission. However, the second antimicrobial choice was effective against mycoplasma in $50(49 \%)$ patients, the third in 34 $(33 \%)$ and the fourth in $3(3 \%)$. Overall, 12 (12\%) patients were treated with macrolides, 48 (47\%) with fluoroquinolones, 21 (20\%) with doxy- or tetracycline and $16(16 \%)$ with a combination of effective antimicrobials. Six $(6 \%)$ patients were discharged without treatment effective against M. pneumoniae.

\section{Discussion}

The effect of M. pneumoniae as a respiratory pathogen has long been debated. In previous literature, M. pneumoniae was usually described to cause mild and self-limiting infections [6]. M. pneumoniae has also been suggested to be a common finding in the upper respiratory tract of asymptomatic individuals [11]. In this case series of mycoplasma PCR-positive cases, the patients had quite severe symptoms. In addition to fever and cough, shortness of breath was a common symptom of M. pneumoniae infection even in previously healthy young adults. Nasal symptoms were rare.

Data on the clinical picture of M. pneumoniae infection, especially in adults, are limited and conflicting. The most common respiratory symptoms of $M$. pneumoniae infection in this series and in the literature are cough and fever [6], and the lack of nasal symptoms found here and in one previous study in adults is an important clinical note [12]. Unexpectedly, the subjective feeling of dyspnoea or shortness of breath was common in our patients. According to the 2012 Cochrane review [13], wheezing is less common in mycoplasma patients compared with other community-acquired pneumonias. However, afterwards, Medjo et al. found wheezing to be typical in mycoplasma infection [14]. Another study consisting of children indicated that symptoms were similar between M. pneumoniae and respiratory virus infections [15]. In this series, the clinical picture of M. pneumoniae infection differed from that of viral respiratory infections.

About $5 \%$ of our patients required intensive care unit admission. This is consistent with the study of Khyory et al. [3], which found that $16 \%$ of patients with M. pneumoniae infection required intensive care unit admission and highlighted the role of $M$. pneumoniae as a competent pathogen that could cause severe infections. In general, the incidence of pneumonia is the highest among older adults, and underlying illnesses are present in almost $80 \%$ of the patients [16]. In this series of M. pneumoniae PCR-positive patients, most of the cases presented in previously healthy young adults without specific risk factors, and the symptoms were not affected by underlying diseases. The most common underlying disease in our series was asthma, which was present in $8 \%$ of the patients. This rate is almost equal to the prevalence of asthma in the Finnish general population, and thus, previous asthma seems to not be a risk factor for $M$. pneumoniae infection [17].

Several changes to the antimicrobial therapy were needed in most patients, as their condition did not improve until receiving an antibiotic effective against $M$. pneumoniae. The treatment guidelines in Finland do not endorse the use of tetracyclines, macrolides or fluoroquinolones as the first-line treatment for community-acquired pneumonia. Thus, only $10 \%$ received an antibiotic effective against $M$. pneumoniae as the first empiric choice. Only six patients were discharged without effective treatment. Most of the patients went through several changes in their medication, which probably postponed their recovery and prolonged their hospital stay. According to the Cochrane review concluded in 2015, there was not enough evidence to make a specific conclusion about the antibiotic treatment of M. pneumoniae infection [9]. Placebo-controlled trials have been suggested $[9,18,19]$. However, our results indicated that it might not be ethically acceptable to perform placebo-controlled trials in M. pneumoniae infections, at least in a hospital-based setting.

In children at least, about $30-60 \%$ of lower respiratory infections have been found to be mixed infections [8, 16, 20,21]. In our patients, most infections were isolated M. pneumoniae infections, and only eight (10\%) patients were detected to have a respiratory virus. Moreover, M. pneumoniae was the only bacterium found in multiplex PCR. As the childhood general vaccination with conjugated pneumococcal vaccine has decreased the incidence of classical pneumococcal pneumonia, the proportion of M. pneumoniae as the aetiology of CAP in children is increasing [20]. This may also gradually occur among adolescents and adults.

The epidemics of $M$. pneumoniae have been shown to occur mostly in late fall $[4,15]$. During this study period, no M. pneumoniae epidemic occurred in the area. When the 3 study years were summarised, the incidence seemed to have peaked at late fall even outside epidemics.

A major limitation of this study is its retrospective case analysis design. Thus, the indications for the samples were not necessarily consistent throughout the population, which may have affected for example the age distribution in this 
study. The information collected from the medical records is always incomplete. Some symptoms (i.e. shortness of breath) were recorded as a subjective report from the patients, limiting the reliability of the results. Moreover, this study was hospitalbased and did not include patients treated at primary care.

In conclusion, M. pneumoniae can cause severe infections that require hospitalisation and an effective antimicrobial treatment. Pneumonia with cough and dyspnoea without nasal symptoms are clinical signs for clinicians to warrant the PCR test for mycoplasma and to consider the empiric antibiotic covering of M. pneumoniae.

Authors' contributions Riku Metsälä and Solja Ala-Korpi collected the data, performed the analyses and wrote the first draft of the manuscript. Juha Rannikko and Merja Helminen critically revised the manuscript. Marjo Renko designed and supervised the work and revised the manuscript. All authors accepted the final form of the manuscript.

Funding Open Access funding provided by University of Eastern Finland (UEF) including Kuopio University Hospital. This is an investigator-driven study with no external funding or support.

\section{Declarations}

Ethics and informed consent As a register based study, consent to participate or approval of the ethics committee was not applicable and the study was performed with the permission of the authorities of the local hospital.

Conflict of interest The authors declare no conflict of interest.

Open Access This article is licensed under a Creative Commons Attribution 4.0 International License, which permits use, sharing, adaptation, distribution and reproduction in any medium or format, as long as you give appropriate credit to the original author(s) and the source, provide a link to the Creative Commons licence, and indicate if changes were made. The images or other third party material in this article are included in the article's Creative Commons licence, unless indicated otherwise in a credit line to the material. If material is not included in the article's Creative Commons licence and your intended use is not permitted by statutory regulation or exceeds the permitted use, you will need to obtain permission directly from the copyright holder. To view a copy of this licence, visit http://creativecommons.org/licenses/by/4.0/.

\section{References}

1. Meyer Sauteur P, van Rossum A, Vink C (2014) Mycoplasma pneumoniae in children: carriage, pathogenesis, and antibiotic resistance. Curr Opin Infect Dis 27(3):220-227

2. Waites KB, Xiao L, Liu Y, Balish MF, Atkinson TP (2017) Mycoplasma pneumoniae from the respiratory tract and beyond. Clin Microbiol Rev 30(3):747-809

3. Khoury T, Sviri S, Rmeileh AA, Nubani A, Abutbul A, Hoss S et al (2016) Increased rates of intensive care unit admission in patients with Mycoplasma pneumoniae: a retrospective study. Clin Microbiol Infect 22(8):711-714

4. Kurkela S, Puolakkainen M, Hokynar K, Nieminen T, Saxen H, Mannonen L et al (2019) Mycoplasma pneumoniae outbreak, Southeastern Finland, 2017-2018: molecular epidemiology and laboratory diagnostic lessons. Eur J Clin Microbiol Infect Dis 38(10):1867-1871

5. Meyer Sauteur PM, Unger WWJ, Nadal D, Berger C, Vink C, van Rossum AMC (2016) Infection with and carriage of Mycoplasma pneumoniae in children. Front Microbiol 7:329

6. Koichi I, Kinichi I, Takahiro T, Kosuke K, Yoshitomo M, Shigeki $\mathrm{N}$ et al (2014) Clinical features, risk factors and treatment of fulminant Mycoplasma pneumoniae pneumonia: a review of the Japanese literature. J Infect Chemother 20:181-185

7. Narita M (2010) Pathogenesis of extrapulmonary manifestations of Mycoplasma pneumoniae infection with special reference to pneumonia. J Infect Chemother 16(3):162-169

8. Rodrigues CMC, Groves H (2018) Community-acquired pneumonia in children: the challenges of microbiological diagnosis. J Clin Microbiol 56(3)

9. Samantha G, John G, Anne C (2015) Antibiotics for communityacquired lower respiratory tract infections secondary to Mycoplasma pneumoniae in children (Review). Cochrane Database Syst Rev 1:CD004875

10. Kuntaliitto. Sairaanhoitopiirit 2018. Available at: https://www. kuntaliitto.fi/sites/default/files/media/file/Ervat Sairaanhoitopiirit2018.pdf

11. Spuesens E, Fraaij P, Visser E, Hoogenboezem T, Hop W, Aichem $\mathrm{L}$ et al (2013) Carriage of Mycoplasma pneumoniae in the upper respiratory tract of symptomatic and asymptomatic children: an observational study. PLoS Med 10(5):e1001444

12. Miyashita N, Kawai Y, Kato T, Tanaka T, Akaike H, Teranishi H et al (2016) Rapid diagnostic method for the identification of Mycoplasma pneumoniae respiratory tract infection. J Infect Chemother 22(5):327-330

13. Wang K, Gill P, Perera R, Thomson A, Mant D, Harnden A (2012) Clinical symptoms and signs for the diagnosis of Mycoplasma pneumoniae in children and adolescents with community-acquired pneumonia. Cochrane Database Syst Rev 10:CD009175

14. Medjo B, Atanaskovic-Markovic M, Radic S, Nikolic D, Lukac M, Djukic S (2014) Mycoplasma pneumoniae as a causative agent of community-acquired pneumonia in children: clinical features and laboratory diagnosis. Ital J Pediatr 40(1):104

15. Søndergaard MJ, Friis MB, Hansen DS, Jørgensen IM (2018) Clinical manifestations in infants and children with Mycoplasma pneumoniae infection. PLoS One 13(4):e0195288

16. Jain S, Williams DJ, Arnold SR, Ampofo K, Bramley AM, Reed C et al (2015) Community-acquired pneumonia requiring hospitalization among U.S. children. N Engl J Med 372(9):835-845

17. Koponen P, Borodulin K, Lundqvist A, Sääksjärvi K, Koskinen S (ed) (2018) Terveys, toimintakyky ja hyvinvointi Suomessa - FinTerveys 2017-tutkimus. http:/urn.fi/URN:ISBN:978-952-343-105-8

18. Eliakim-Raz N, Robenshtok E, Shefet D, Gafter-Gvili A, Vidal L, Paul M et al (2012) Empiric antibiotic coverage of atypical pathogens for community acquired pneumonia in hospitalized adults. Cochrane Database Syst Rev 2012(9):CD004418

19. Foshaug M, Vandbakk-Rüther M, Skaare D, Grude N, Lindbæk M (2015) Mycoplasma pneumoniae detection causes excess antibiotic use in Norwegian general practice: a retrospective case-control study. Br J Gen Pract J R Coll Gen Pract 65(631):e82-e88

20. Chiu C, Chen C, Wong K, Tsai M, Chiu C, Huang Y (2013) Impact of bacterial and viral coinfection on mycoplasma pneumonia in childhood community-acquired pneumonia. J Microbiol Immunol Infect 48(1):51-56

21. Juvén T, Mertsola J, Waris M, Leinonen M, Meurman O, Roivainen M et al (2000) Etiology of community-acquired pneumonia in 254 hospitalized children. Pediatr Infect Dis J 19(4):293-298

Publisher's note Springer Nature remains neutral with regard to jurisdictional claims in published maps and institutional affiliations. 